\title{
How should we overcome the threat by the pandemic of 2019-nCoV? Epidemic simulation using the SIRS model
}

\author{
Jun Inamo ${ }^{1}$ \\ Received: 10 March 2020 / Revised: 24 March 2020 / Accepted: 1 April 2020 / Published online: 9 May 2020 \\ (C) International League of Associations for Rheumatology (ILAR) 2020
}

Since the first identification in December 2019 in Wuhan, China, a number of cases of novel coronavirus (2019-nCoV)infected patients is increasing around the world. It has been reported that there are high-risk populations with high mortality, such as elderly people and those with comorbidities [1]. However, no specific treatment has been developed yet. Historically, humans have overcome the pandemics of viral infection by preventing secondary infection. Several studies have simulated the spread of $2019-\mathrm{nCoV}$, and the basic reproduction number $\left(R_{0}\right)$ ranges from 2.2 to $3.6[2,3]$. On the other hand, patients treated with disease-modifying antirheumatic drugs may have insufficient antibody response, implying the possibility of reinfection [4].

Here, to simulate the efficacy of preventing secondary infection in population with consideration of reinfection, I simulated a scenario of one person with 2019$\mathrm{CoV}$ infection entering into a population of 1000 people with the hypothesis that a part of them lose immunity. I used the SIRS model which is composed of different categories within a population; the susceptible $(S)$, infected $(I)$, recovered $(R)$, and returned-susceptible $(S)$ compartments, respectively. The model can be written as the following ordinary differential equation:

Electronic supplementary material The online version of this article (https://doi.org/10.1007/s10067-020-05083-7) contains supplementary material, which is available to authorized users.

Jun Inamo

inamo.z2@keio.jp

1 Division of Rheumatology, Department of Internal Medicine, Keio University School of Medicine, 35 Shinanomachi, Shinjuku-ku, Tokyo 160-8582, Japan

$$
\begin{gathered}
\frac{d S}{d t}=-\frac{\beta S I}{N}+\xi R \\
\frac{d I}{d t}=\frac{\beta S I}{N}-\gamma I \\
\frac{d R}{d t}=\gamma I-\xi R \\
R 0=\frac{\beta}{\gamma}
\end{gathered}
$$

where $S, I$, and $R$ are proportions of susceptible, infected, and recovered individuals, respectively, $\beta$ is the transmission rate of infection, $\gamma$ is recovery rate (inverse of the infectious period), and $\xi$ is returning rate of recovered individuals to the susceptible statue due to loss of immunity. $N(=S+I+R)$ is the total population. The simulation was started with $S=999, I=1$, and $R=0$ for the following 100 days after the entry of one person with 2019-nCoV. Considering the uncertainty of parameters in the real-world outside China, I combined parameters based on considerable circumstances. Analyses were performed by $\mathrm{R}$ software.

First, parameters were set as $(\beta, \gamma, \xi)=(0.5,1 / 20,0.01)$ based on assumption that infectious duration is 20 days [5] and $1 \%$ of recovered individuals return to the susceptible statue due to loss of immunity. As $\beta$ decreased $0.5,0.4$, 0.3, 0.1, and 0.05 (Fig. 1 a, b, c, d, e, and f, respectively), the maximum number of infected people decreased and the peak delayed, suggesting the effectivity of decreasing transmission rate by standard precautions. When $\beta$ was 0.05 , the secondary infection did not occur. $R_{0}$ in each situation was $10,8,6,4,2$, and 1 , respectively. Next, to evaluate the efficacy of prompt isolation of infected patients, $\gamma$ was stratified with $1 / 20,1 / 16,1 / 12,1 / 8,1 / 4$, and $1 / 1$ with keeping $\beta N$ and $\xi$ the same. As a result, the maximum number of infected people decreased as $\gamma$ increased (Fig. 2 a, b, c, d, e, and f, respectively). The secondary infection did not occur in the case of $\gamma=1 / 1 . R_{0}$ in each situation was $10,8,6,4,2$, and 1 , respectively. These trends were the same as the case $\xi=0$ (Supplementary 
SIRS model

a $\beta=0.5, \gamma=0.05, \rho=0.01$

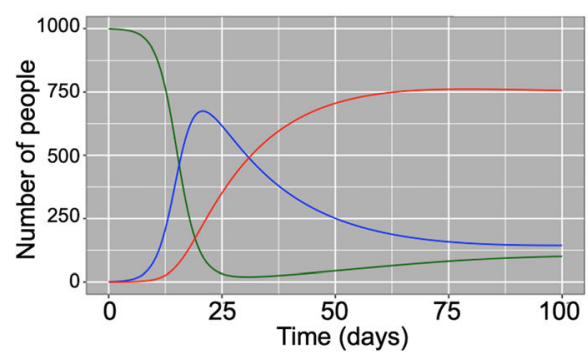

d SIRS model

d $\beta=0.2, y=0.05, \rho=0.01$

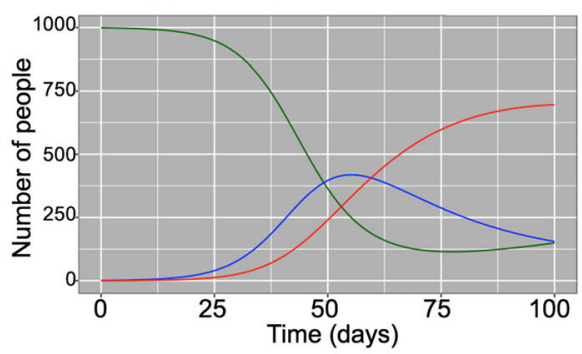

b SIRS model

$\beta=0.4, \gamma=0.05, \rho=0.01$

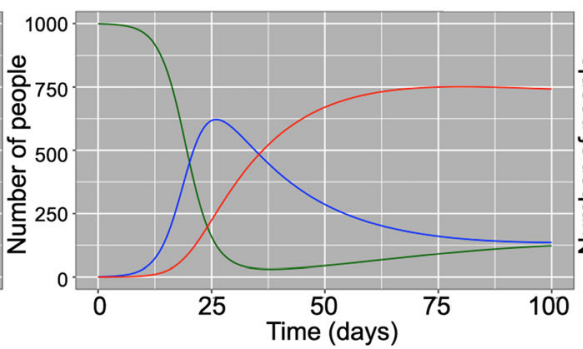

SIRS model

e $\beta=0.1, \gamma=0.05, \rho=0.01$

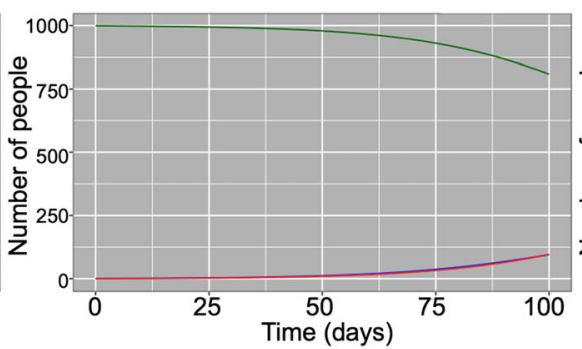

SIRS model

$\beta=0.3, \gamma=0.05, \rho=0.01$

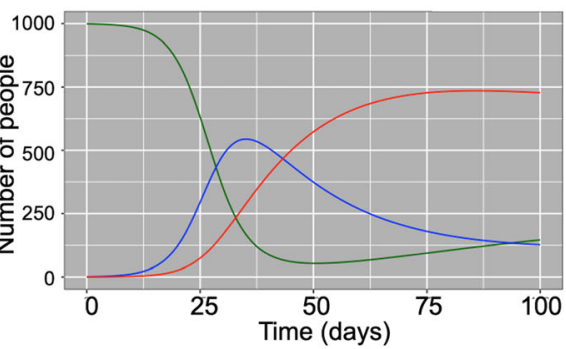

f SIRS model

$\beta=0.05, \gamma=0.05, \rho=0.01$

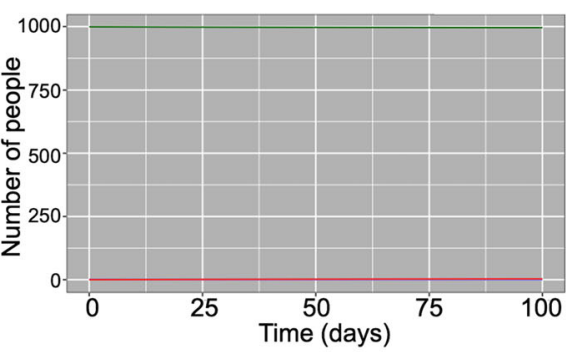

- Susceptible - Infected - Recovered

Figure 1 SIRS model for assessing impact of transmission rate $(\beta)$. Transmission rate were stratified with 0.5 (a), 0.4 (b), 0.3 (c), 0.2 (d), 0.1 (e), and 0.05 (f) with keeping recovery rate $(\gamma)$ and returning rate $(\xi)$ as $1 / 20$ and 0.01 , respectively

Figs. 1 and 2; when 2019-nCoV infection would lead to long-lasting immunity).

This study suffers from several limitations. First, since the clinical characteristics of 2019-nCoV are largely unknown, the value of parameters used in this simulation may not be proper. Second, the SIRS model is a simple model based on some assumptions; the population is closed (no vital dynamics such as birth and death) and the course of the infection is short (emergent outbreak). Also, the possibility that asymptomatic patients could be infectious to others is not considered in this model [6]. Third, because the SIR model assumes a homogeneous infection network between individuals, the

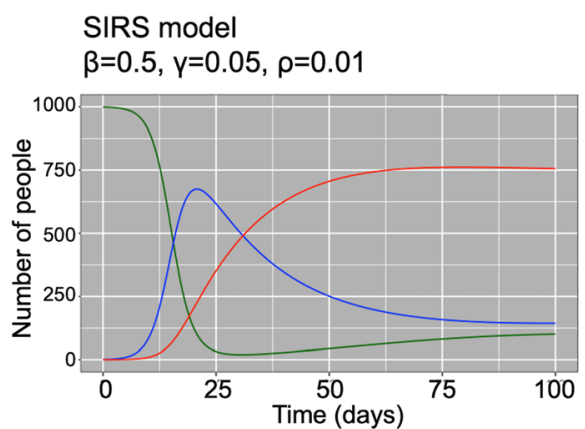

SIRS model

$\beta=0.5, y=0.125, \rho=0.01$

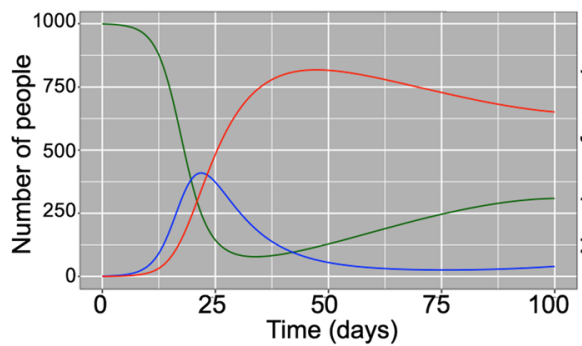

SIRS model

$\beta=0.5, \gamma=0.0625, \rho=0.01$

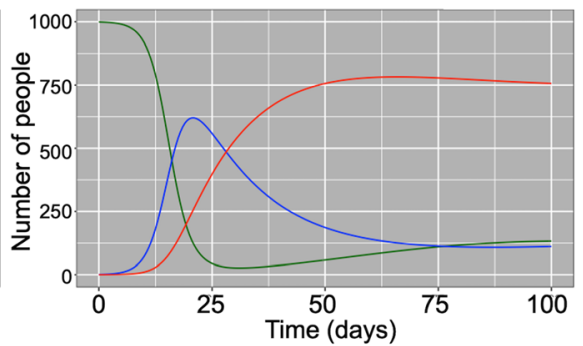

SIRS model

$\beta=0.5, y=0.25, \rho=0.01$

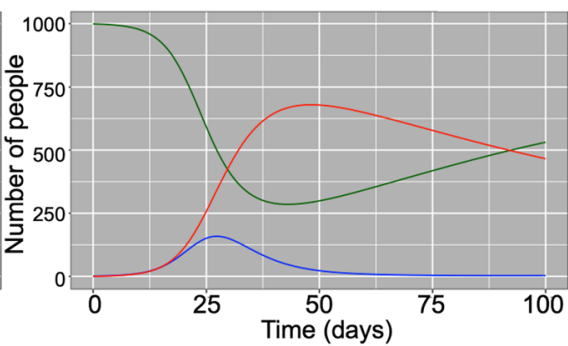

SIRS model

$\beta=0.5, y=0.0833, \rho=0.01$

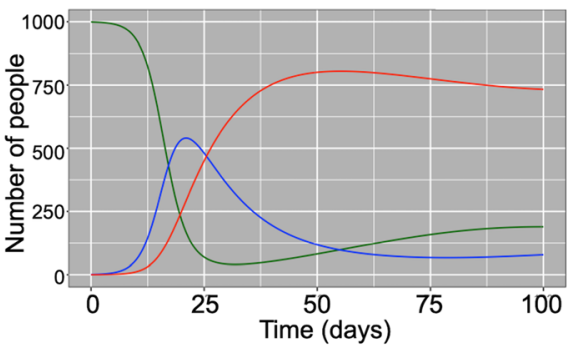

SIRS model

$\beta=0.5, y=1, \rho=0.01$

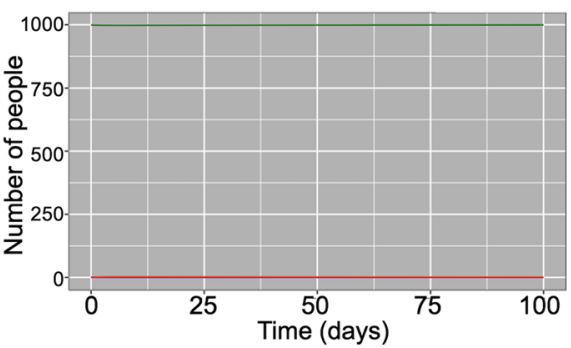

\section{- Susceptible - Infected - Recovered}

Fig. 2 SIRS model for assessing impact of recovery rate $(\gamma)$. Recovery rates were stratified with 1/20 (a), 1/16 (b), 1/12 (c), 1/8 (d), 1/4 (e), and 1/1 (f) with keeping transmission rate $(\beta)$ and returning rate $(\xi)$ as 0.5 and 0.01 , respectively 
heterogeneity of individuals such as disease status was not considered. Forth, other than standard precautions, $\beta$ is also affected by virologic characteristics and factors of hosts. Therefore, the result of this study cannot apply to data in the real-world directly. However, this study demonstrated that outbreaks can be prevented by performing appropriate precautionary measures even in populations with consideration of the possibility of reinfection.

Author's contributions Conceptualization, formal analysis, and writing were conducted by the author.

Data availability All custom computer codes in the generation or processing of the described data are available in the supplementary file.

\section{Compliance with ethical standards}

Ethics approval and consent to participate Ethical approval or individual consent was not applicable.

Consent for publication Not applicable.

Disclosures None.

\section{References}

1. Wu Z, McGoogan JM (2020) Characteristics of and important lessons from the coronavirus disease 2019 (COVID-19) outbreak in China: summary of a report of 72314 cases from the Chinese
Center for Disease Control and Prevention. JAMA. 323:1239. https://doi.org/10.1001/jama.2020.2648

2. Zhao S, Lin Q, Ran J, Musa SS, Yang G, Wang W, Lou Y, Gao D, Yang L, He D, Wang MH (2020) Preliminary estimation of the basic reproduction number of novel coronavirus (2019-nCoV) in China, from 2019 to 2020: a data-driven analysis in the early phase of the outbreak. Int J Infect Dis 92:214-217

3. Li Q, Guan X, Wu P, Wang X, Zhou L, Tong Y, Ren R, Leung KSM, Lau EHY, Wong JY, Xing X, Xiang N, Wu Y, Li C, Chen Q, Li D, Liu T, Zhao J, Liu M, Tu W, Chen C, Jin L, Yang R, Wang Q, Zhou S, Wang R, Liu H, Luo Y, Liu Y, Shao G, Li H, Tao Z, Yang Y, Deng Z, Liu B, Ma Z, Zhang Y, Shi G, Lam TTY, Wu JT, Gao GF, Cowling BJ, Yang B, Leung GM, Feng Z (2020) Early transmission dynamics in Wuhan, China, of novel coronavirus-infected pneumonia. N Engl J Med 382:1199-1207. https://doi.org/10.1056/NEJMoa2001316

4. Hesselstrand R, Nagel J, Saxne T, Geborek P, Skattum L, Kapetanovic MC (2018) Immunogenicity and safety of pneumococcal vaccination in patients with systemic sclerosis. Rheumatology (Oxford) 57:625-630

5. Zou L, Ruan F, Huang M, Liang L, Huang H, Hong Z, Yu J, Kang M, Song Y, Xia J, Guo Q, Song T, He J, Yen HL, Peiris M, Wu J (2020) SARS-CoV-2 viral load in upper respiratory specimens of infected patients. N Engl J Med 382:1177-1179. https://doi.org/10.1056/ NEJMc2001737

6. Rothe C, Schunk M, Sothmann P, Bretzel G, Froeschl G, Wallrauch C, Zimmer T, Thiel V, Janke C, Guggemos W, Seilmaier M, Drosten C, Vollmar P, Zwirglmaier K, Zange S, Wölfel R, Hoelscher M (2020) Transmission of 2019-nCoV infection from an asymptomatic contact in Germany. N Engl J Med 382:970-971. https://doi.org/10. 1056/NEJMc2001468

Publisher's note Springer Nature remains neutral with regard to jurisdictional claims in published maps and institutional affiliations. 